\title{
An Efficient Method of License Plate Location under Complicated Background
}

\author{
Fengsong $\mathrm{Hu}^{1,2}$ \\ 1.College of Information Science and Engineering \\ 2.Postdoctoral of System \\ 1.Hunan University;2.Being Normal University \\ 1.Changsha,China;2.Being,China \\ fshu@hnu.cn
}

\author{
Manman Zhou \\ College of Information Science and Engineering \\ Hunan University \\ Changsha,China \\ 15573184361@163.com
}

\begin{abstract}
License plate location is the key of license plate recognition.In order to locate license plate quickly and accurately under complicated background,this paper presents a new location algorithm.Firstly,we make a set of pretreatment to original image,such as graying,binarization,noise reduction and edge detection.Secondly,use the method of mathematical morphology to get candidate areas of license plate.Finally, make the feature matching in candidate areas which using a method of improved feature matching --SSIFT(Scale-invariant Feature Transform).The experimental results show that, this method has a high recognition efficiency in the complex background and it has good robustness.
\end{abstract}

Keywords-license plate location; edge detection; Mathematical morphology;SSIFT

\section{INTRODUCTION}

The current license plate location algorithm mainly contains the following ones: based on texture feature,based on color feature as in [1],based on mathematical morphology as in [2],based on edge detection as in [3], neural network. These algorithm have efficient location results which only in good surrounding environment such as good light condition,parallel license plate and unbroken license plate.In complex background, these algorithm can not be locate plate accurately.In this paper,it proposes a efficient algorithm which combinate edge detection, mathematical morphology and SSIFT(Simplified Scaleinvariant Feature Transform). Firstly,we make a set of preprocessing to original image,such as graying,binarization,noise reduction and edge detection.Secondly,use the method of mathematical morphology to get candidate areas of license plate .Finally,make the feature matching in candidate areas which using a method of improved feature matching -SSIFT.

The basic process of license plate localization algorithm as shown in the Fig. 1 .

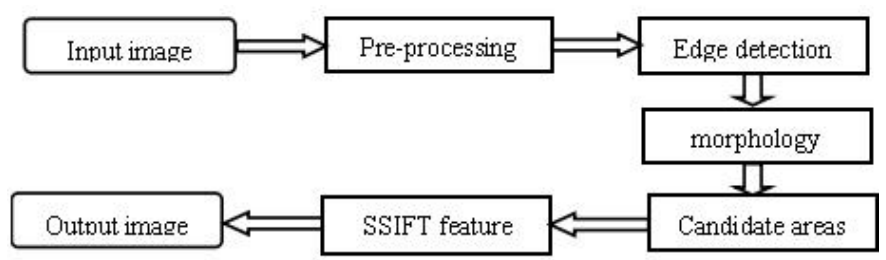

Figure 1. The basic process of license location

\section{IMAGE PRE-PROCESSING}

\section{A. Gray processing}

In order to reduce calculation, this paper firstly make a gray processing to the color image.In the MATLAB, using rgb2gray () function can convert color image to gray image [4].The effect is shown in Fig. 2.

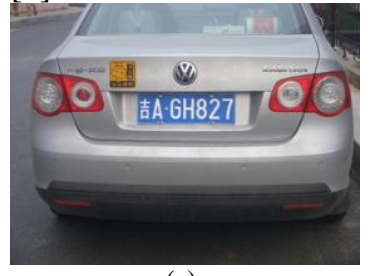

(a)

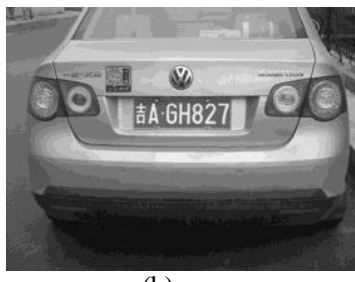

(b)
Figure 2. Gray processing: (a) color image, (b) gray image

\section{B. Denoising}

After gray processing,we need do binarization processing to gray image.In this paper we use the function named cvThreshold().The threshold is set to 100,and the maximum is set to 255. The effect is shown in Fig 3 (a).As the photos taken with a digital camera are easily affected by Gaussian noise, which will bring a lot of difficulties to subsequent image processing,so,in this paper,we adopt the Laplace operator to realize high-pass filter for the purpose of image enhanced and image sharpening [5].

First,make Fig 3 and formula (1) to execute a convolution.

$$
\left[\begin{array}{ccc}
0 & 1 & 0 \\
1 & -4 & 1 \\
0 & 1 & 0
\end{array}\right]
$$


Then,we adopt Laplace operator to enhance image, The concrete syntax as formula (2) shown.

$$
g(x, y)=f(x, y)+c\left[\nabla^{2} f(x, y)\right]
$$

As formula (3) shows, $\mathrm{f}(\mathrm{x}, \mathrm{y})$ index as input image, $\mathrm{g}(\mathrm{x}, \mathrm{y})$ index as enhanced image.If the center coefficient in formula(2) is plus,then set $\mathrm{c}$ as 1,else set $\mathrm{c}$ as -1.In this paper,w set $\mathrm{c}$ as -1 .

$\nabla^{2} \mathrm{f}(x, y)$ is the Laplace, The concrete syntax as formula (3) shown.

$$
\nabla^{2} f(x, y)=\frac{\partial^{2} f(x, y)}{\partial x^{2}}+\frac{\partial^{2} f(x, y)}{\partial y^{2}}
$$

After the enhanced of image by Laplace operator,the effect is shown in Fig 3(b).

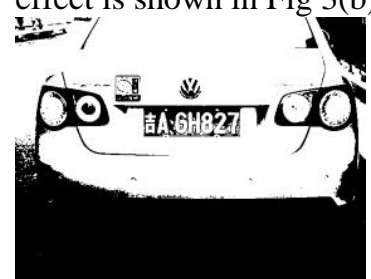

(a)

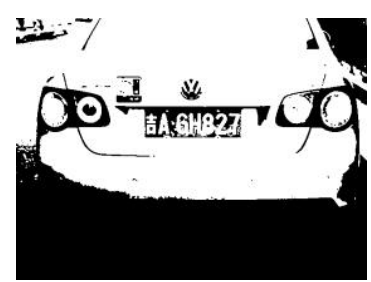

(b)
Figure 3. The denoising: (a) binarization, (b) image enhanced

\section{Edge detecting}

Edge detection can highlight the edges of the image, weaken the outside edge of the image area.In the car image, license plate area is rich in edges, which contains the horizontal edges, the vertical edges and oblique edges.But in the areas out of License plate, it has less edges[6].So we can use this feature to separate license and background areas.After repeated comparison and testing,we find that Robert operator in $2 \times 2$ has the best effect.Denoising processing lay a good foundation to edge detecting.For this reason ,Robert operator become the most suitable for our edge detecting. The concrete syntax of Robert is shown as formula (4).

$$
g(x, y)=\left\{[\sqrt{f(x, y)}-\sqrt{f(x+1, y+1)}]^{2}+[\sqrt{f(x, y)}-\sqrt{f(x-1, y-1)}]^{2}\right\}^{1 / 2}
$$

As formula (4) shows, $\mathrm{g}(\mathrm{x}, \mathrm{y})$ index as input image which has integer pixel coordinates.In this paper we use two $2 \times 2$ convolution kernel $\left(\left[\begin{array}{cc}1 & 0 \\ 0 & -1\end{array}\right]\left[\begin{array}{cc}1 & 0 \\ 0 & -1\end{array}\right]\right)$ as the Robert operator.Each point in the input image are convolved with the two convolution kernel. The effect is shown in Fig. 4

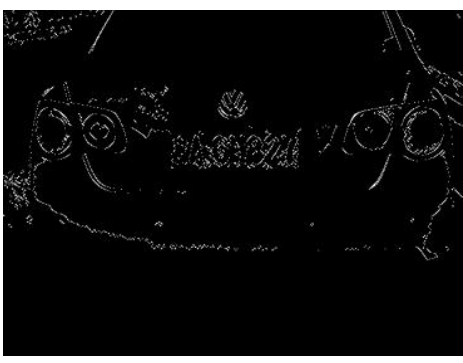

Figure 4. Edge detecting

\section{CANDIDATE AREAS}

\section{A. Mathematical morphology}

Mathematical morphology is a mathematical method for analyzing the geometry and structure.The basic operation is corrosion, swelling, opening and closing. Since the size of license plate chang largely with different camera angles,in this paper,we use the closing operator which is a horizontal one-dimensional structural element.Assume the preprocessing image as $\mathrm{A}_{0}$, and structural element as $\mathrm{B}_{1 \times \mathrm{n}}$ [7].The processing of Mathematical morphology is as follows.

$$
A_{1}=A_{0} \cdot B_{1 \times n}=\left(A \otimes B_{1 \times n}\right) \Theta B_{1 \times n}
$$

$$
A_{2}=A_{1} \circ B_{1 \times(n-1)}=\left(A_{1} \Theta B_{1 \times(n-1)}\right) \oplus B_{1 \times(n-1)}
$$

Formula (5) represent transverse closure operation,it reduce the complexity of computation computation and avoid the enlarging of longitudinal interference.Formula (6) adopt a mend of opening operator based on the decrease of the size of lateral dimension.After the operator of formula (5)and(6),we get the effect as Fig 5.

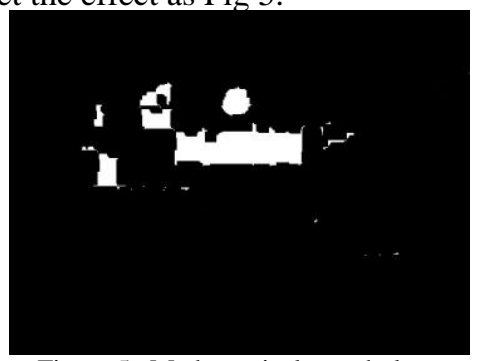

Figure 5. Mathematical morphology

\section{B. The extracting of rectangle}

According to the geometry texture Characteristics of the plate, the length-width ratio of rectangular area can not be too big.The normal length-width ratio of license plate is 3.14 ,but for the reason of shooting angle and the tilting of plate,the length-width ratio is about 2.2 3.8.If meet the above conditions ,it should be the candidate area.After the extracting of rectangle,we get the effect as Fig 6. 


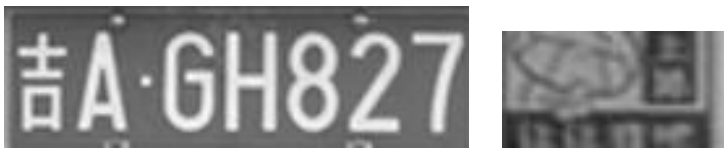

Figure 6. Candidate areas

\section{ACCURATE POSITIONING OF LICENSE PLATE}

The license plate of our country is composed of Chinese characters, digits, uppercase letters. letters. So we can use the character feature of license plate for feature matching. As Chinese character has a lot of strokes,and it is more complicated to other characters,so we can get more stable match points.For these reasons, we adopt the first character of license plate--Chinese character to do the feature match. In fact, this process is the using of SSIFT feature to do feature match among two images [8].

\section{A. SSIFT}

1) Extrema detection of scale-space[9]: Scale space of an image is defined as a function named $\mathrm{L}(\mathrm{x}, \mathrm{y}, \sigma)$.It is the convolution of Gaussian function $\mathrm{G}$ and the image I:

$$
\begin{aligned}
& L(x, y, \sigma)=G(x, y, \sigma) * I(x, y) \\
& G(x, y, \sigma)=\frac{1}{2 \pi \sigma^{2}} e^{-\left(x^{2}+y^{2}\right) / 2 \sigma^{2}}
\end{aligned}
$$

Firstly,we build the DOG(Difference-ofGaussian)pyramid of the image and then make extrema detection in DOG scale-space.As formula (9) shows, $\mathrm{D}(\mathrm{x}, \mathrm{y}, \sigma)$ is a difference between the two adjacent images.

$$
D(x, y, \sigma)=(G(x, y, k \sigma)-G(x, y, \sigma)) * I(x, y)=L(x, y, k \sigma)-L(x, y, \sigma)
$$

If one point is the maximum or minimum in the layer of DOG scale space,we suppose this point as the matching point.

\section{2) Form a feature vector}

a) After the formation of multi-scale feature points, feature points as the center of a circular form is needed to determine the scope of the neighborhood statistics (see Fig 7), the window size using Lowe recommended $9 \sigma \times 9 \sigma$, thus setting the radius of the circular windows as $4.5 \sigma$ in which make statistics to the 12 gradient directions [10].

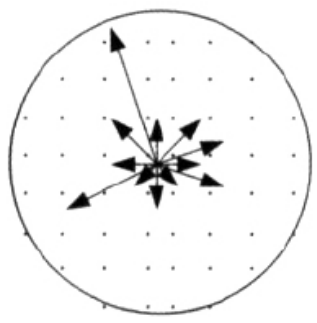

Figure 7. Round Statistics window b) Make normalized processing to these 12 gradient directions, and make sure the invariability of illumination.Assuming $\mathrm{D}$ is a characteristic vector of a feature point. $D=\left(d_{1}, d_{2} \ldots, d_{12}\right)$. After we make normalized processing to $\mathrm{D}$, the effect is shown as formula (10).

$$
\bar{D}=\frac{D}{\sqrt{\sum_{i=1}^{12} d_{i}^{2}}}=\left(\bar{d}_{1}, \bar{d}_{2} \ldots, \bar{d}_{12}\right)
$$

c) If $\bar{d}_{1}=\max \left\{\bar{d}_{i}, \bar{d}_{i} \in \bar{D}\right\}$, then the final characteristic vector is shown as formula(11),else turn to the fourth step.

$$
\bar{D}=\left(\bar{d}_{1}, \bar{d}_{2}, \bar{d}_{3}, \bar{d}_{4}, \bar{d}_{5}, \bar{d}_{6}, \bar{d}_{7}, \bar{d}_{8}, \overline{d_{9}}, \overline{d_{10}}, \overline{d_{11}}, \overline{d_{12}}\right)
$$

d)Make a left cycle and move the entire vector until the statistics of a maximum gradient direction move to the first element of the vector.Suppose $d_{5}$ as the maximum element of the vector,then the final characteristic vector is shown as formula(12).

$$
\bar{D}=\left(\overline{d_{5}}, \overline{d_{6}}, \overline{d_{7}}, \overline{d_{8}}, \overline{d_{9}}, \overline{d_{10}}, \overline{d_{11}}, \overline{d_{12}}, \overline{d_{1}}, \overline{d_{2}}, \overline{d_{3}}, \overline{d_{4}},\right)
$$

3) Feature matching:In order to guarantee the stability of the matching effect, adding another more matching in the original algorithm.We should record the matching coordinates in the first matching, Then exchange sequence of matching image,and match them again,until we get the same result twice.

\section{B. The processing of license plate matching}

The application of SSIFT to license plate location has a good effect.The principle and the process is as follows:Firstly,by using the SSIFT feature matching algorithm we can calculate the characteristics of the candidate region.Then, match with the Chinese characters in the template library of Chinese character,and choose the most matching point of the Chinese characters as the matching results. The template library of Chinese characters in this paper only contains the first character of license plate,with fewer features, fast matching speed.Use this template and the candidate region to match, we can eliminate the effect of rotation, scaling and stretching of the license plate image, and it can lay a good foundation for the recognition of next.

\section{THE EXPERIMENTAL RESULTS}

Since by using MATLAB less efficient when processing cycle, in order to improve program efficiency, the SSIFT feature matching is implemented by the mixed programming which use MATLAB and $\mathrm{C}$. In practical 
algorithm, in order to prevent the information of the license plate image from losing, the candidate region of the plate located by a mathematical morphology extend about 5 percent in four directions of up, down, left and right. The Picture is the Rendering which is matched between characters in the Chinese template library and candidate region of the license plate .Because Chinese characters have succeed matched with the first character of the plate, it is possible to exclude the candidate area in Fig8 the interference region.

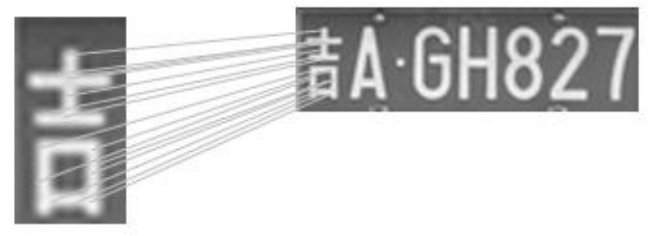

Figure 8. Feature matching of SSIFT

\section{CONCLUSION}

TABLE I. ANALYSIS OF EXPERIMENTAL DATA

\begin{tabular}{|c|c|c|c|}
\hline $\begin{array}{l}\text { Total } \\
\text { number } \\
\text { of } \\
\text { images }\end{array}$ & $\begin{array}{c}\text { Numbers } \\
\text { of correct }\end{array}$ & $\begin{array}{c}\text { Numbers of } \\
\text { error }\end{array}$ & $\begin{array}{c}\text { Recognition } \\
\text { rate }\end{array}$ \\
\hline 300 & 289 & 11 & $96 \%$ \\
\hline
\end{tabular}

In this paper, we do some research in the license plate location under complex background, and propose a combination of edge detection, mathematical morphology and SSIFT quickly locate feature matching algorithm which is applied to a collection of 300 images whose size are 640 $\times 480$ experimental.The results show that after applying edge detection and mathematical morphology into locating the license plate of the candidate region, it is feasible that SSIFT feature matching algorithm is applied to license plate location and it can improve recognition speed and accuracy.It can also solve bad problems in the feature matching algorithm of license plate recognition effect and improve the usefulness of the algorithm.

\section{REFERENCES}

[1] Cui Zongyong,Xie mei. A Method for Blue Background White Characters Cars License Plate Location [C] //Computer Science and Information Technology,2009:393-395.

[2] Li Gang,Zeng Ruili,Lin Ling. A License Plate Location Method Based on Mathematical Morphology [J] // Chinese Journal of Scientific Instrument ,2007,28(7):1323-1327.

[3] Rongbao Chen,Yunfei Luo.An Improved License Plate Location Method Based On Edge Detection [C] //Applied Physics and Industrial Engineering,2012:1350-1356

[4] Cuiyuan Jiang,Zhengxiang Wang,Xianghu Wang.A Location System Based On Mathematical Morphology [J] // Chinese Journal of Scientific Instrument,2011,32(6):36-39

[5] Xiongfei Liu,Qiaoyun Long,Gen Li.License Plate Localisation Algorithm Based On Line Scanning Method and Wavelet Transform [J] //Computer Applications and Software,2013,30(5):279-282.

[6] Junfei Zhuo,Yi Hu.Research of License Plate Locating Method Based On Edge Detective and Projection [J] // Bulletin of Science and Technology,2010,26(3):438-441

[7] Wei Chen.Approach for License Plate Location Based on Mixed Features under Complex Background [J] // Modern Computer,2011,6(04):35-38

[8] Tao Yang,Senlin Zhang.Approach of Vehicle Plate Extraction Based on HSV Color Space and SIFT feature [J] // Application Research of Computers2011,7(15):20-23.

[9] LOWE D G. Object Recognition Form Local Scale-invariant Features [C] // International Conference on Computer Vision,1999:1150-1157.

[10] Li Liu,Fuyuan Peng,Kun Zhao.Simplified SIFT Algorithm for Fast Image matching [J] //Infrared and Laser Engineering,2008,37(1):181184. 